\title{
Septicemia por Klebsiella Enterobacter en recién nacidos
}

Drs.: Pedro Herskovic, Verónica Geldres, Juan P. Beca, Jorge Howard y Clara Román.*

Entre las etiologías de los procesos infecciosos bacterianos neonatales diagnosticados en el Centro de Prematuros del Hospital Luis Calvo Mackenna, llama la atención observar que en los últimos años el $\mathbf{5 3 , 5 \%}$ de los casos de septicemia han sido causados por gérmenes del grupo Klebsiella Enterobacter. ${ }^{1}$ La septicemia neonatal por este germen no sólo fue más frecuente, sino también más grave y tuvo mayor letalidad.

La alta incidencia y mayor gravedad motivó la revisión de los casos recientes de septicemia neonatal por Klebsiella Enterobacter del Centro de Prematuros. Se hizo un análisis clínico y un estudio complementario de sensibilidad a antibióticos, con el fin de conocer mejor este cuadro clínico y sus alternativas terapéuticas.

\section{MATERIAL Y METODO}

Se revisaron las fichas clínicas de 28 recién nacidos hospitalizados en el Centro de Prematuros del Hospital Luis Calvo Mackenna en el período comprendido entre septiembre de 1974 y junio de 1977, en los que se hizo el diagnóstico de septicemia por Klebsiella Enterobacter. El diagnóstico se fundamentó en la existencia de un cuadro clínico compatible y la comprobación de uno o más hemocultivos positivos a ese germen.

El grupo estudiado incluyó 18 recién nacidos de pretérmino y 10 de término; 12 fueron de sexo masculino y 16 de sexo femenino. La edad gestacional promedio del grupo fue de 35,7

*Centro de Prematuros, Hospital Luis Calvo Mackenna. semanas (rango 26 a 41 semanas). El peso de nacimiento fue en promedio de $2.099 \mathrm{~g}$. (rango 900 a $3.750 \mathrm{~g}$.).

En cada caso se analizaron los datos de su procedencia, los antecedentes del parto, la edad, el día de hospitalización en que se hizo el diagnóstico, el cuadro clínico que lo hizo sospechar, las características del hemograma (tomado en un lapso no mayor de $\mathbf{4 8}$ horas del momento en que se obtuvo el primer hemocultivo positivo) y el resultado terapéutico.

El estudio complementario de sensibilidad a los antibióticos se realizó en 38 cepas de Klebsiella Enterobacter, aisladas de cultivos de secreción rectal en igual número de recién nacidos hospitalizados en el Servicio, en los meses de diciembre de 1976 y junio de 1977 . Se estudió la sensibilidad a Gentamicina, Tobramicina, Kanamicina, Cotrimexazol, Amikacina y Ampicilina, utilizando el método de dilución en placa de agar. ${ }^{2}$

\section{RESULTADOS}

La procedencia de los 28 recién nacidos fue muy variada, ya que fueron enviados de hospitales del S.N.S. de Santiago y provincias, de clínicas privadas de Santiago, e incluso algunos llegaron de sus domicilios. En la mayoría de los casos el diagnóstico se hizo dentro de los tres primeros días de hospitalización $(64,3 \%)$. (Tabla 1.)

Los antecedentes del parto revelaron que en sólo 9 casos éste fue eutócico $(32,1 \%)$, en 12 fue por cesárea $(42,8 \%), 2$ fueron domiciliarios, en uno hubo aplicación de fórceps, uno fue inducido por maniobras abortivas y en 3 casos no se pudo obtener este antecedente. 


\section{Tabla 1}

Día de hospitalización en que se diagnosticó septicemia por Klebsiella Enterobacter.

Dias de

\begin{tabular}{lrc} 
hospit. & N. $^{\circ}$ & $\%$ \\
\hline 1 a 3 & 18 & 64,3 \\
4 a 6 & 4 & 14,3 \\
7 y más & 6 & 21,4 \\
\hline Total & 28 & 100,0 \\
\hline
\end{tabular}

La rotura de membranas ocurrió dentro de las 24 horas previas al parto en 20 casos $(95,2 \%)$. En sólo uno, este lapso fue mayor de 24 horas. En 7 casos no se pudo obtener este antecedente.

Las manifestaciones clínicas que hicieron sospechar el diagnóstico de septicemia fueron, en orden de frecuencia: mal estado general, escleredema, hepatoesplenomegalia, dificultad respiratoria, ictericia, palidez terrosa, crisis de apnea, distensión abdominal, disminución de la tolerancia alimentaria, púrpura, diarrea y artritis. (Tabla 2.)

Tabla 2

Manifestaciones clínicas iniciales en 28 casos de septicemia neonatal por Klebsiella

Enterobacter.

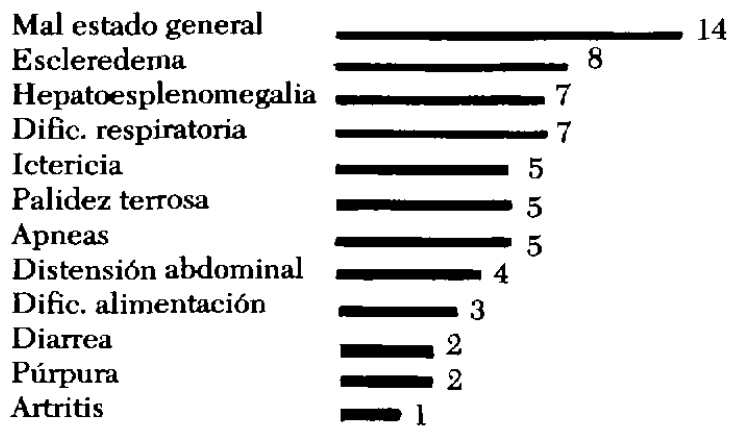

Fue posible obtener hemograma en un lapso de 48 horas antes o después del momento en que se tomó el primer hemocultivo positivo solamente en 18 casos. Del hemograma se analizó el recuento de neutrófilos por $\mathrm{mm}^{3}$, el recuento de baciliformes y la apreciación de la cantidad de plaquetas al frotis, aspectos considerados de utilidad para apoyar el diagnóstico de infección, según diversos autores. ${ }^{3,4,5}$

El recuento de neutrófilos se encontró por sobre o debajo de los límites establecidos por Gregory y $\mathrm{Hey}^{3}$ para las diferentes edades en 8 casos $(44,4 \%)$. El recuento de baciliformes estuvo sobre los límites de la tabla de Altman y Dittmer $^{6}$ para cada edad en 8 niños $(44,4 \%)$. Las plaquetas se encontraron disminuidas al frotis en 9 casos $(50 \%)$. (Tabla 3.)

Tabla 3

Alteraciones del hemograma en 18 casos de septicemia neonatal por Klebsiella Enterobacter
Alteración
N. ${ }^{\circ} \%$

Neutrofilia y

plaquetas dism.

15,6

al frotis

Neutropenia y

plaquetas dism.

al frotis

$2 \quad 11,1$

Neutrofilia

$5 \quad 27,8$

Plaquetas dism.

6

al frotis

Desviación a

$2 \quad 11,1$

izquierda

\begin{tabular}{lrr} 
Sin alteraciones & 2 & 11,1 \\
\hline Total & 18 & 100,0 \\
\hline
\end{tabular}

El $88,8 \%$ de los casos tuvo alteraciones significativas del hemograma al menos en uno de los parámetros analizados. En la mitad de estos casos el hemograma reveló más de una alteración sugerente de infección.

Los hechos clínicos que potencialmente podrían haber actuado como factores desencadenantes de la septicemia en algunos casos, fueron: cateterización umbilical prolongada para administración de fleboclisis (2 casos), parto inducido por maniobra abortiva ( 1 caso), cirugía mayor ( 5 casos) y exsanguíneo-transfusión ( 1 caso).

La letalidad de la septicemia por Klebsiella Enterobacter en este grupo de recién nacidos fue de $46,4 \%$. Cuando el diagnóstico se hizo en los primeros cuatro días de vida, falleció el 72,7\% de los pacientes, mientras la septicemia diagnos- 
ticada después de los cuatro días de vida tuvo una letalidad de $29,4 \%$. (Tabla 4.)

\section{Tabla 4}

Letalidad de la septicemia neonatal por

Klebsiella Enterobacter según la edad al momento del diagnóstico.

Edad de diagnóstico N..$^{\circ}$ Muertes Letalidad (días)

\begin{tabular}{cccc}
\hline 1 a 4 & 11 & 8 & $72,7 \%$ \\
5 y más & 17 & 5 & $29,4 \%$ \\
\hline Total & 28 & 13 & $46,4 \%$ \\
\hline
\end{tabular}

El tratamiento antibacteriano de la septicemia fue iniciado en 27 de los 28 casos con la asociación de Penicilina ( $100.000 \mathrm{U}$ por $\mathrm{Kg}$. de peso por día) o uno de sus derivados y Gentamicina (5 a $7 \mathrm{mg}$. por $\mathrm{Kg}$. de peso por día). El caso restante fue tratado con la asociación de Rifampicina y Cotrimexazol, por haber recibido durante 10 días, y hasta 4 días antes del diagnóstico de septicemia, Penicilina y Gentamicina por una Bronconeumonia. Ocho de los casos tratados con Penicilina o derivados y Gentamicina fallecieron antes de completar 48 horas de tratamiento. Reci- bieron más de 48 horas de tratamiento 19 niños, de los cuales 10 mejoraron $(52,6 \%$ ) y en 9 hubo fracaso terapéutico $(47,4 \%)$. Los fracasos terapéuticos incluyeron 5 niños que fallecieron y 4 que mejoraron después de cambiar antibióticos. (Tabla 5.)

\section{Tabla 5}

Resultado terapéutico con Penicilina o derivados y Gentamicina en 27 casos de septicemia neonatal por Klebsiella Enterobacter.

\begin{tabular}{lr} 
Resultado & N. $^{\circ}$ \\
\hline Muertos antes de 48 hrs. & 8 \\
Mejoría clínica & 10 \\
Fracaso terapéutico & 9 \\
\hline Total & 27 \\
\hline
\end{tabular}

El estudio complementario de sensibilidad a antibióticos de las 38 cepas de Klebsiella Enterobacter demostró que el $31,6 \%$ era sensible a Gentamicina, el 10,5\% a Ampicilina, el 26,3\% a Tobramicina, el $36,8 \%$ a Kanamicina, el 52,6\% a Cotrimexazol y el 94,7\% a Amikacina. (Tabla 6.)

Tabla 6

\section{Sensibilidad a antibióticos de 38 cepas de Klebsiella Enterobacter aisladas en el Centro de Prematuros en diciembre de 1976 y junio de 1977.}

\begin{tabular}{cccccc} 
Gentamicina & Tobramicina & Kanamicina & Cotrimexazol & Amikacina & Ampiciliıa \\
$-5 \mathrm{mcg} / \mathrm{ml}$ & $-5 \mathrm{mcg} / \mathrm{ml}$ & $-12,5 \mathrm{mcg} / \mathrm{ml}$ & $-12,5 \mathrm{mcg} / \mathrm{ml}$ & $-12,5 \mathrm{mcg} / \mathrm{ml}$ & $-12,5 \mathrm{mcg} / \mathrm{ml}$ \\
\hline
\end{tabular}

\begin{tabular}{|c|c|c|c|c|c|c|}
\hline $\begin{array}{l}\text { N.o de cepas } \\
\text { estudiadas }\end{array}$ & 38 & 38 & 38 & 38 & 38 & 38 \\
\hline $\begin{array}{l}\text { N.o de cepas } \\
\text { sensibles }\end{array}$ & 12 & 10 & 14 & 20 & 36 & 4 \\
\hline $\begin{array}{l}\text { \% de cepas } \\
\text { sensibles }\end{array}$ & 31,6 & 26,3 & 36,8 & 52,6 & 94,7 & 10,5 \\
\hline
\end{tabular}

\section{DISCUSION}

La septicemia neonatal constituye un cuadro relativamente frecuente y de alta letalidad en el Centro de Prematuros del Hospital Luis Calvo Mackenna. Se ha demostrado que las infecciones bacterianas en recién nacidos son producidas en un alto porcentaje por los gérmenes que los colonizan antes de las 24 horas de vida. ${ }^{7}$ Como los niños de esta serie procedían de diversas maternidades y en la mayoría de los casos la septicemia se demostró durante los tres primeros días de hospitalización, es posible suponer que el problema de infecciones por Klebsiella Entero- 
bacter sea similar en otros establecimientos hospitalarios del país.

Entre los antecedentes de los recién nacidos que presentaron septicemia por Klebsiella Enterobacter, llama la atención el gran número de ellos que fue producto de parto distócico, lo que es considerado como factor de riesgo de infección neonatal. ${ }^{8}$ La rotura prematura de membranas no fue un factor causal importante de infecciones en este estudio, ya que en un solo caso ésta ocurrió a más de 24 horas antes del parto. La cateterización umbilical prolongada para administración de fleboclisis representa un riesgo importante de infección, porque el catéter puede ser puerta de entrada de gérmenes patógenos. ${ }^{9}$ En los casos en que se practicó cirugía mayor o exsanguíneo-transfusión, el rol desencadenante de la infección de estos hechos fue poco claro, ya que ésta estaba probablemente establecida antes de estos procedimientos.

Las manifestaciones clínicas que hicieron sospechar el diagnóstico fueron generalmente cambios sutiles, frecuentemente advertidos inicialmente por el personal de enfermería, tales como leve compromiso del estado general, disminución de la tolerancia alimentaria, distensión abdominal, etc... Si se esperan signos o síntomas más categóricos de infección grave, es probable que se formule tardíamente el diagnóstico y que las posibilidades terapéuticas sean por lo tanto menores.

El hemograma en esta serie constituyó un recurso de diagnóstico útil, ya que en el $88,8 \%$ de los casos presentó alteraciones de importancia, al ser analizados desde diversos puntos de vista, tales como recuento de neutrófilos, recuento de baciliformes y apreciación de plaquetas al frotis, presentándose todas estas alteraciones con frecuencias similares.

Es posible que la frecuencia con que se encontró cada una de las alteraciones hubiera sido mayor de haberse contado con hemogramas realizados en forma seriada. Sin embargo la interpretación adecuada del hemograma en recién nacidos requiere aplicar tablas que consideren las grandes variaciones fisiológicas normales de los valores hematológicos en el período neonatal.

Los resultados obtenidos en esta serie concuerdan con el concepto de la utilidad del hemograma en el diagnóstico de las infecciones graves neonatales.
La letalidad fue mayor en los casos de septicemia que dieron manifestaciones clínicas precoces que en aquellas de aparición tardía. Esto se explica probablemente porque los casos más graves se expresan clínicamente en forma más precoz y con mayor severidad. Apoya este concepto el hecho que entre los casos de septicemia de manifestación clínica precoz hubo 4 en los cuales se obtuvo hemocultivos positivos en el primer día de vida y todos ellos fallecieron.

La alta letalidad de la septicemia por Klebsiella Enterobacter en esta serie y los malos resultados que hemos observado con el tratamiento empleado, hacen plantear la necesidad de buscar otras alternativas de terapia antibacteriana que ofrezcan mejores posibilidades que las que aparentemente ofrece la Gentamicina en este momento. Según los resultados del estudio complementario de sensibilidad a antibióticos, la Amikacina parece ser más promisoria, aunque el número de cepas estudiadas aún es pequeño.

En conclusión, la septicemia neonatal por Klebsiella Enterobacter constituye un cuadro relativamente frecuente en nuestro medio y con una letalidad de casi la mitad de los casos. Para mejorar su pronóstico, es necesario intentar diagnósticos más precoces, extremar las medidas terapéuticas tendientes a mejorar las condiciones del huésped y seleccionar el esquema antibacteriano más adecuado de acuerdo con los estudios periódicos de sensibilidad.

\section{RESUMEN}

Se analizan los datos clínicos de 28 recién nacidos de ténmino y de pretérmino con septicemia por Klebsiella enterobacter hospitalizados en el Centro de Prematuros del Hospital L. Calvo Mackenna entre setiembre 1974 y junio 1977. Se realizo además un estudio complementario de sensibilidad de 38 cepas de Klebsiella Enterobacter aisladas en secreción rectal de recién nacidos hospitalizados en el Centro de Prematuros en los meses de diciembre de 1976 y junio de 1977.

Las manifestaciones clínicas iniciales más frecuentes fueron cambios sutiles como compromiso del estado general, escleredema, hepatoesplenomegalia, dificultad respiratoria $y$ otros.

El hemograma constituyó un recurso de apoyo diagnóstico útil en el $88,8 \%$ de los casos, demostrando una o más de las alteraciones siguientes: neutrofilia, neutropenia, aumento de baciliformes y plaquetas disminuidas al frotis.

La letalidad global de la serie fue $46,4 \%$. El tratamiento antibacteriano se realizó con la asociación de Penicilina y Gentamicina en 27 de los 28 casos. Ocho de ellos fallecieron antes de las 48 horas de tratamiento; de los 19 casos tratados por más de 48 horas hubo mejoría clínica en 10 y fracaso terapéutico en 9 casos.

El estudio complementario de sensibilidad de 38 cepas de Klebsiella Enterobacter demostro: $31,6 \%$ de cepas sensibles 
a Gentamicina, $36,8 \%$ a Kanamicina, $52,6 \%$ a Cotrimexazol, y $94,7 \%$ de las cepas fueron sensibles a Amikacina.

Se plantea que para mejorar el pronóstico de la septicemia neonatal por Klebsiella Enterobacter es necesario hacer diagnósticos más precoces, extremar las medidas tendientes a mejorar las condiciones del huésped y seleccionar el esquema antibacteriano más adecuado de acuerdo con estudios periódicos de sensibilidad.

\section{SUMMARY}

An analysis was performed of the clinical information pertaining to 28 premature and full term newbom infants in whom the clinical and bacteriological diagnosis of KlebsiellaEnterobacter sepsis was made and who were hospitalized at the Centro de Prematuros, Hospital Luis Calvo Mackenna between September 1974 and June 1977. A concomitant study of the bacterial sensitivities of 38 strains of Klebsiella-Enterobacter isolated from rectal swabs of newboms and prematures hospitalized in the months of December 1976 and June 1977 was also carried out.

The initial signs and symptoms were subtle changes in the general condition, scleredema, enlargement of the liver and spleen, respiratory distress and others.

In $88 \%$ of the patients the blood count was helpfill in making the diagnosis by showing one or more of the following: neutrophilia, neutropenia, increased percentage of immature white blood cells and diminished amount of platlets seen on the periphereal smear.

Total mortality was $46.4 \%$. A combination of penicillin and gentamicin was used as antibiotic treatment in 27 of the 28 infants.

Eight patients died before 48 hours of treatment had been completed; of the remaning 19 cases 10 improved with this treatment and 9 were considered failures of treatment.

The antibiotic susceptibility study of the 38 strains of
Klebsiella-Enterobacter showed that $31.6 \%$ were sensitive to gentamicin, $36.8 \%$ to kanamycin, $52.6 \%$ to cotrimexazol and $94.7 \%$ to amikacin.

The following conclussions could be extracted from this study: to improve the prognosis of Klebsiella-Enterobacter sepsis an early diagnosis and improvement of the newborns general condition is paramount. The treatment should be with the least toxic and most effective antibiotic selected according to the antibiotic susceptibility studies performed in each nursery.

\section{REFERENCIAS}

${ }^{1}$ Herskovic P., Toso A., Beca J.P., Donoso E. Etiología de las infecciones bacterianas neonatales. Rev. Chile Ped. (En prensa.)

2 Hostalin K.C., Markley A.N., Woodman E. Agar plate dilution method for routine antibiotic susceptibility testing in a hospital laboratory. Am. J. Clin. Path. 60: 384, 1973.

${ }^{3}$ Gregory J., Hey E. Blood neutrophil response to bacterial infections in the first month of life. Arch. Dis. Childh. 47: $747,1972$.

${ }^{4}$ Xanthou M. Leucocyte blood picture in ill newborn babies. Arch. Dis. Childh. 47: 741, 1972.

5 Akenzua G., Hui Y., Milner R., Zipursky A. Neutrophil and band counts in the diagnosis of neonatal infections. Pediatrics 54: 38, 1974.

6 Neonatology, diseases of the fetus and infant. Edited by Behrman R. The C.V. Mosby Company, Saint Louis, 1973.

${ }^{7}$ Beca J.P., Filippa R., Donoso E. Infección bacteriana en el recién nacido de bajo peso: colonización, personal y ambiente. Rev. Chile Ped. 48: 34, 1977.

${ }^{8}$ Gluck L., Wood H., Fousek M. Septicemia of the newborn. Pediat. Clin. N. Amer. 13: 1131, 1966.

${ }^{9}$ Krauss A., Albert R., Khunan M. Contamination of umbilical catheters in the newborn infant. J. Pediat. 77: $965,1970$. 